\title{
Some features of rare and endangered plant species introduction
}

\author{
Tatyana Elisafenko* and Olga Dorogina \\ Central Siberian Botanical Garden of the SB RAS, Zolotodolinskaya Str., 101, Novosibirsk, 630090, \\ Russia
}

\begin{abstract}
The article discusses the features and main stages of rare and endangered plant species introduction. It is necessary to be guided by the principles of priority and expediency in the preparatory stage, which includes the analysis of literary data and preliminary studies in nature. The first stage is the primary introduction; it involves a detailed study of natural populations and organizing an introduction site. Based on the obtained results, the acclimatization assessment is determined. The study of biological features including reproduction is carried out at the second stage. The third stage includes investigations of the introduced populations. The phenorhythm stability, limiting factors determining dates of phenophase onset, the introduction population optimal size and number, seed longevity and conditions of their storage, self-maintenance ability are revealed. As a result, it is given the adaptation assessment, and recommendations are developed to save rare and endangered species in nature and under introduction conditions. Additional studies include applying karyological, molecular-genetic, biotechnological techniques, and possibilities to reconstruct natural populations.
\end{abstract}

Since the mid XX century, a prior activity trend of introduction centers has been creating a collection or exposition of rare and endangered plant species. The International Botanic Gardens Plant Conservation Program [1] presents ways to develop own biodiversity conservation programs and tools for botanical gardens. The priority is the biodiversity conservation and rational use in the region of the introduction center location. The complete conservation of plant biodiversity involves applying different methods, and the governmental, public organizations and interested entrepreneurs cooperation.

Introducing rare and endangered plant species applies traditional techniques, however, the there are some peculiarities - it takes more time and investments, and the results are often negative. At present, it is necessary to develop guidelines for the gradual study and preservation of rare and endangered plant species in the introduction centers. Researches should be aimed to study the biological and physical processes affecting the biodiversity diminution, as well as to identify its causes, and to develop tools for the regional biodiversity conservation and rational. The introduction as a measure of conservation turns out to be inexpedient for some of these species. Otherwise, introductions may be the only way to preserve other species.

\footnotetext{
* Corresponding author: tveli@list.ru
} 
The work objective is to develop guidelines for such species introduction related to the relevance of rare and endangered species conservation, studying their biological features, identifying useful properties.

Preparatory and primary introduction are important steps in introducing rare and endangered species. The preparatory stage includes the object selection, where the main principles are priority and expediency [2]. Besides, it is necessary to carry out a preliminary population study at this stage, because the low abundance and insignificant knowledge of this object hamper to introduce and reproduce rare and endangered species. Comprehensive expeditionary research, including specialists of various profiles (florists, geobotanists, taxonomists, biomorphologists, introductors) will provide a fruitful search for populations, valid habitat characterization, the population state description and receiving the material. The introduction of rare and endangered species populations should begin with studying the natural population: its condition assessment, including demographic indicators [3, 4], plant vitality [5], ontogenesis and reproductive characteristics. It is necessary to be guided by the program for studying the species of red-books lists.

The obtained herbarium, field and literature data are analyzed and the introduction forecast is made based on some approaches: the ecological-historical analysis by M.V. Kultiasov, the flora genetic technique of choosing introduced species by K.A. Sobolevskaya, the generic complexes method by F.N. Rusanov, the tool of accounting the acclimatization experience in the past by A.N. Avrorin, the biomorph comparison by M.T. Mazurenko, the phytocenotic one by R.A. Karpisonova. Highly specialized and stenotopic species are unpromising with high probability. However, only a direct experiment can reveal the introduction success of a particular species, as the interspecific competition elimination, natural habitats imitation, agrotechnical measures may be sufficient conditions for plant growth and development. The preparatory stage is resulted in the introductory passport, which includes the data of the natural initial population: a geographical location, habitat topography, date, collector, habitat phytocenotic and ecological description, plant community species composition (estimate the species participation in it), evaluating a population state, demographic indicators, viability plants, data on ontogenesis and reproductive characteristics study.

The first stage includes the material search and selection, assessment of the plant primary introduction (acclimatization), reproduction and study of introducent biological features. Diaspores can be obtained from nature or introduction centers. The latters may contain genotypes significantly different from natural populations. Therefore, the refinement of the selected species systematic position is mandatory, if necessary, identification is carried out by molecular-genetic methods. Particular attention is paid to the high representativeness and maintenance of the taxa genetic purity in developing the methodology of rare species reproduction and preservation in vitro. Therefore, one of the most important stages of rare species and genotype preservation is identifying the initial and micropropagated plants by ISSR markers. Some researchers consider that rare and endangered species introduction by seeds obtained by delectus from other regions is unacceptable. Such species introduction should be immaculately clean, using seeds from natural populations for this purpose [6]. It is also a controversial statement that rare species introduction should be carried out only by seed, because it is necessary to exclude plants removal from nature. In fact, living plant removal in limited quantities from plural populations is permissible, as well as vegetatively mobile plants. An important issues is the representativeness of the rare and endangered species population under conditions of introduction. The recommended optimal area and number of introduced populations are the following: $100 \mathrm{~m}^{2}$ (5-10 individuals) for trees, $50 \mathrm{~m}^{2}$ (10-30 individuals) for shrubs, $40 \mathrm{~m}^{2}$ (100-500 individuals) for large grasses, $10-20 \mathrm{~m}^{2}$ (500-1000 individuals) for small-shrub and non-roselatte herbs [6]. 
Expositions and collections of rare and endangered plant species should be organized according to an ecological principle creating sites with microenvironmental conditions for mesophytes, xerophytes, petrophytes, hygrophytes, etc. Rare and endangered plant species can be represented both introducing populations (in the presence of a large number of individuals and changes in generations), and samples consisting of several individuals. Every variant is realized as monoculture, or as a phytogroup part. Monoculture plots are usually supported by agrotechnical measures (weeding, thinning, loosening, fertilizer), this allows maintaining plants with good vitality and producing seed every year. Not all rare and endangered species can exist in monoculture conditions, it's necessary to create phytogroups (areas imitating plant communities) for some of them. It is advisable to form phytogroups for educational activities as various micro-exposures. However, dense planting of different species will increase competitiveness, allelopathy may occur, therefore, seed productivity decrease will take place. In addition, if the activity of the introduction center is carried out as part of the global task of introducing rare and endangered species to preserve species diversity, it is advisable to create and maintain hybrid populations. Hybrid populations are individuals of the same species from different points of its range. This is especially true for cross-pollinated plants of the same species grown in a small area without isolation. Hybrid populations increase the probability of successful existence under introduction conditions, and they can be used as donors to reconstruct the disappeared and small populations. During the first stage, the species systematic identity is verified, plants survival rate (the number of introducents survived of the seedlings number), winter hardiness, drought tolerance is determined. The following data should be added to the existing introduction certificate: date of introduction, number of individuals or seeds, location on the introduction site.

The second stage includes introducents' reproduction and their study. It reveals the ability to seed and vegetative reproduction; defines seed germination feature; describes ontogenesis, biological characteristics of growth and development; evaluated the primary introduction, which reflects plants acclimatization to the introduction center conditions.

The third stage is studying the introduced populations. It reveals the stability phenorhythm, limiting factors determined the dates of phenophase onset, an optimal size and number of the introduced population, seeds longevity, their storage conditions, selfmaintaining ability; elaborates recommendations to preserve rare and endangered species in nature and under the conditions of introduction. The main study directions at this stage are to determine the possibility of introduced species (seed or vegetative) reproduction and adaptation assessment. The result is compiling a biological introducing passport of a species, which includes the research results of three stages: name (Latin and Russian), synonyms, categories of rarity according to different lists of the Red Books, species range and habitat (on literary sources), ecological group, presence of herbarium specimens in herbaria of Russia, species locus classicus, photos of herbarium and natural specimens, morphobiological characteristics in nature (on literary data and original research), life form, seed collecting time, collector name, morphology of fruits and seeds: length, width, weight, germ characteristics, seed reproduction features - germination conditions (light, darkness , positive high and low temperatures, their effect duration stage-by-stage), period before germination, period of germination, germination, durability, especially fruiting, percentage of fruit production, seed production, self-seeding ability, dates and methods of sowing seeds, features of ontogenesis, rhythmological group, diseases, pests of introduced plants, survival rate (the ratio of planted individuals and definitive individuals number), agrotechnical measures applied to introduced species, assessment of the species economic properties, adaptation degree of plants, recommended measures to this plant species conservation. 
Creating introductory collections allows carrying on complimentary research: karyological (number of chromosomes), phytochemical (biological active substances of many rare and endangered species are not identified), molecular-genetic, biotechnological ones. The ISSR-marking method has recently been used in population and taxonomic studies, as ISSR markers have a large variability. A modified SDS electrophoresis method in polyacrylamide gel of seed storage proteins $[7,8]$ and a technique of genomic DNA inter-microsatellite regions with ISSR markers [9] can successfully analyze intra- and interpopulation variability and genotyping of individual samples. This study will help to identify adaptation processes occurring at the genetic level, determine the magnitude of genetic variability, control genetically the formation of rare and endemic species stable populations at different stages, and develop recommendations for their conservation. Evaluating the genotype frequency and identifying the most adapted genotypes, which are the basis for the formation of populations in different years, can be carried out on the basis of an annual analysis of electrophoretic spectra of seed storage proteins in populations growing under the introduction conditions. Biotechnological approach application offers fundamentally new opportunities to preserve and reproduce the plant gene pool. Despite the importance of maintaining genetic diversity in natural ecosystems (in situ), it has recently become clear that many rare species cannot be preserved without developing ex situ technologies. An important step is to research plant morphogenesis in vitro using light and scanning electron microscopy, as well as create a crop bank in vitro, and optimize conditions for its maintaining.

\section{Acknowledgements}

The study is carried out according to of the state assignment of the Central Siberian Botanical Garden of the Siberian Branch of the Russian Academy of Sciences, state registration no. AAAA-A17-117012610051-5 and applying bioresource scientific "Collection of Living Plants in Open and Closed Ground" USU 440534.

\section{References}

1. W. Jackson, L.A. Sutherland, International Agenda for Botanic Gardens in Conservation (Botanic Gardens Conservation International, U.K, 2000)

2. T.V. Elisafenko, O.V. Dorogina, Problems of industrial botany in advanced industrial regions (Kemerovo, 2018).

3. L.A. Zhukova, Journal of general biology, 44, 3 (1983)

4. L.A. Zhivotovsky, Rus J of Ecology, 1 (2001)

5. Yu.A. Zlobin, Principles and methods of studying coenotic plant populations (Kazan, 1989)

6. G.P. Semenova, Rare and endangered flora species of Siberia: biology and protection (Novosibirsk, 2007)

7. U.K. Laemmli, Nature, 4 (1970)

8. O.V. Agafonova (Dorogina), A.V. Agafonov, Dep. All-Russian Institute of Scientific and Technical Information, 2467-B91 (1991)

9. H. Nybom, Mol. Ecology, 13 (2004) 